\title{
Chronic Rate Dependent Exit Block After Pulmonary Vein Isolation
}

\author{
Parth Makker ${ }^{1}$, Eric Dulmovits ${ }^{2}$, and Stuart Beldner ${ }^{3}$ \\ ${ }^{1}$ Lenox Hill Hospital \\ ${ }^{2}$ North Shore University Hospital \\ ${ }^{3}$ Northwell Health
}

October 20, 2020

\begin{abstract}
Rate dependent exit block across the pulmonary veins has been previously described immediately following catheter ablation. We report the case of rate dependent PV block seen at repeat ablation 7 years after the index procedure. To our knowledge, this is the first report of chronic rate dependent exit block (i.e. Rate dependent exit block discovered 7 years after circumferential PV antral isolation). We believe that, as with CTI ablation, rate independent pulmonary vein isolation should be confirmed.
\end{abstract}

\section{Chronic Rate Dependent Exit Block After Pulmonary Vein Isolation}

Parth Makker MD ${ }^{1}$, Eric Dulmovits MD ${ }^{1}$, Stuart Beldner MD FHRS ${ }^{1}$

Northwell Health - 300 Community Dr, Manhasset, NY 11030

Corresponding Author:

Parth Makker

11 East $12^{\text {th }}$ St, Apt $1 \mathrm{~F}$

New York, NY 10003

The authors have no conflicts of interest or funding to disclose

Word Count: 913 words

\section{Introduction:}

Pulmonary vein (PV) ectopy is understood to represent the bulk of triggers for atrial fibrillation. ${ }^{1}$ Electrical isolation by demonstration of entrance and exit block in these structures is the cornerstone of the catheter based therapy for this arrhythmia. ${ }^{2}$

Rate dependent conduction block is a well described electrophysiological phenomenon. This is often seen physiologically in the normal conduction system (e.g. a long-short inducing a RBBB, or AV Wenckebach at rapid heart rates). Rate dependent block across the cavo-tricuspid isthmus has been well described after catheter ablation of atrial flutter. It is believed to increase the likelihood of recurrence and, consequently, rate independent block has been supported as the end point for this ablation. Rate dependent block across the pulmonary veins has been previously described immediately following catheter ablation. Jacobson et $\mathrm{al}^{3}$ has previously described rate dependent entrance block (LA:PV) and Yagashita et $\mathrm{al}^{4}$ described rate dependent exit block (PV:LA). We report the case of rate dependent PV block seen at repeat ablation 7 years after the index procedure. 


\section{Case:}

Our patient is a 61 year old man with a history of hypertension and symptomatic paroxysmal atrial fibrillation who underwent pulmonary vein. He remained symptom free with no evidence of recurrence of atrial fibrillation for 7 years following his initial ablation procedure. He then presented with persistent atrial fibrillation complicated by congestive heart failure and severely reduced left ventricular ejection fraction. He underwent electrical cardioversion and his left ventricular systolic function subsequently improved back to normal. A stress test revealed no evidence of ischemia. Given the hypothesis of a tachycardia mediated cardiomyopathy, and limitations of AV nodal blockers in the setting of profound sinus bradycardia, the decision was made to proceed with electrophysiologic testing and repeat ablation despite this single recurrence. Three dimensional electroanatomic mapping (CARTO 3, Biosense Webster, Diamond Bar, CA) of the left atrium suggested persistent isolation with voltage $<.1 \mathrm{mV}$ (entrance block) of the left and right sided pulmonary veins. Pacing revealed exit block within the right sided PVs. The left sided PVs showed exit block at paced cycle length of $600 \mathrm{~ms}$ with capture of the sleeve. At a paced cycle length of $1000 \mathrm{~ms}$, there was conduction to the left atrium, albeit with significant delay (Figures 1 and 2).

We identified the site of electrical breakthrough in the LSPV using escape mapping. ${ }^{5}$ We then proceeded to re-isolate the PV and were subsequently unable to provoke non-pulmonary vein triggers, with and without Isuprel. We then proceeded to isolate posterior wall which demonstrated automaticity with exit block postablation.

\section{Discussion:}

Although rate dependent block is believed to increase the likelihood of recurrent arrhythmia for CTI dependent atrial flutter, it is unlikely to be the culprit for our patient's recurrence of atrial fibrillation. Specifically, the inability to conduct at faster cycle lengths would suggest that automaticity, or tightly coupled premature atrial contractions, would not likely reach the left atrium and thus not trigger arrhythmia. Nonetheless, we did re-isolate the vein.

To our knowledge, this is the first report of chronic rate dependent exit block (i.e. Rate dependent exit block discovered 7 years after circumferential PV antral isolation). The most probable explanation is phase 3 block or tachycardia induced block secondary to the ablation. Normal cells are characterized by a more negative resting potential, larger action potential amplitude, and fast depolarization sodium current. Diseased cells have a less negative resting potential, smaller action potential amplitude of shorter duration, and a much slower depolarizing current that could still be carried by a sodium current with depressed kinetics ${ }^{6}$. The injured tissue has not completely repolarized, and thus cannot generate an action potential at short cycle lengths (i.e. there is a long effective refractory period).

While it is possible this reconnection in the LSPV was the etiology of arrhythmia recurrence, as we were not able to provoke triggers, it is not our hypothesis as the etiology for the recurrent arrhythmia. The electrophysiologic observation is interesting and may have more significant implications in the acute setting. We have seen the use of adenosine in PV isolation as well as the importance of entrance and exit block. We now observe rate dependent block of a pulmonary vein. We believe that, as with CTI ablation, rate independent pulmonary vein isolation should be confirmed.

Figure 1: Capture of LA at paced CL of $1000 \mathrm{~ms}$ from inside the LSPV 


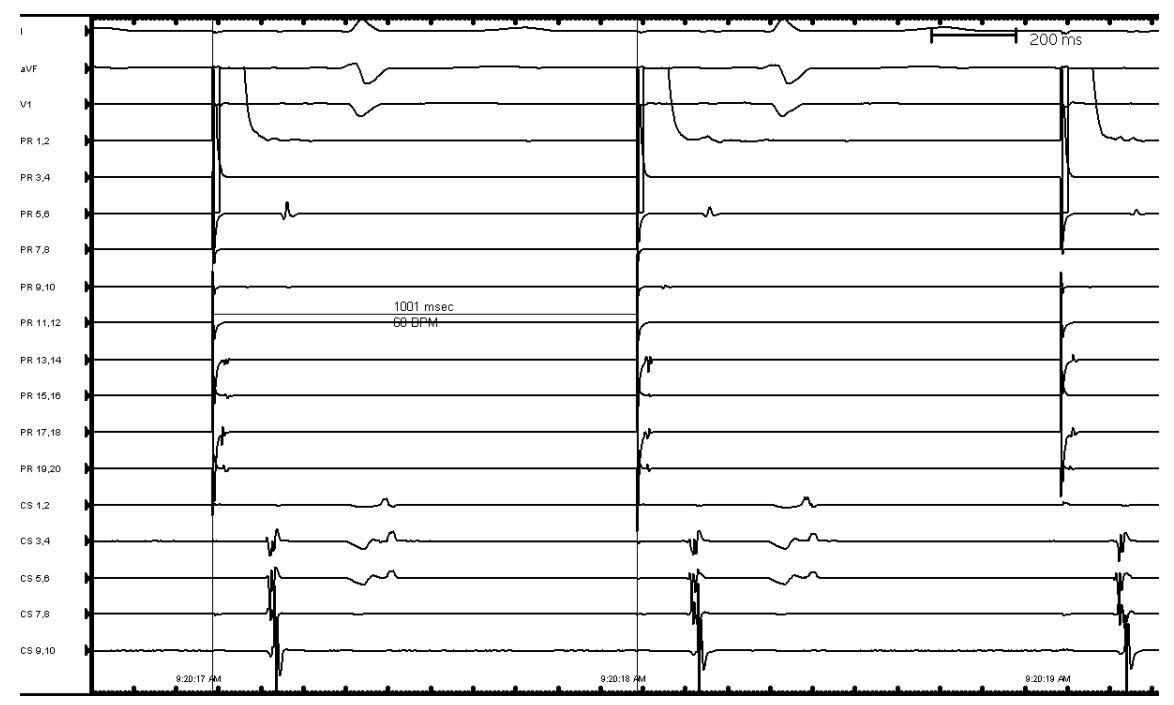

Figure 2: Capture of LSPV sleeve at paced CL of $600 \mathrm{~ms}$ with exit block into the LA

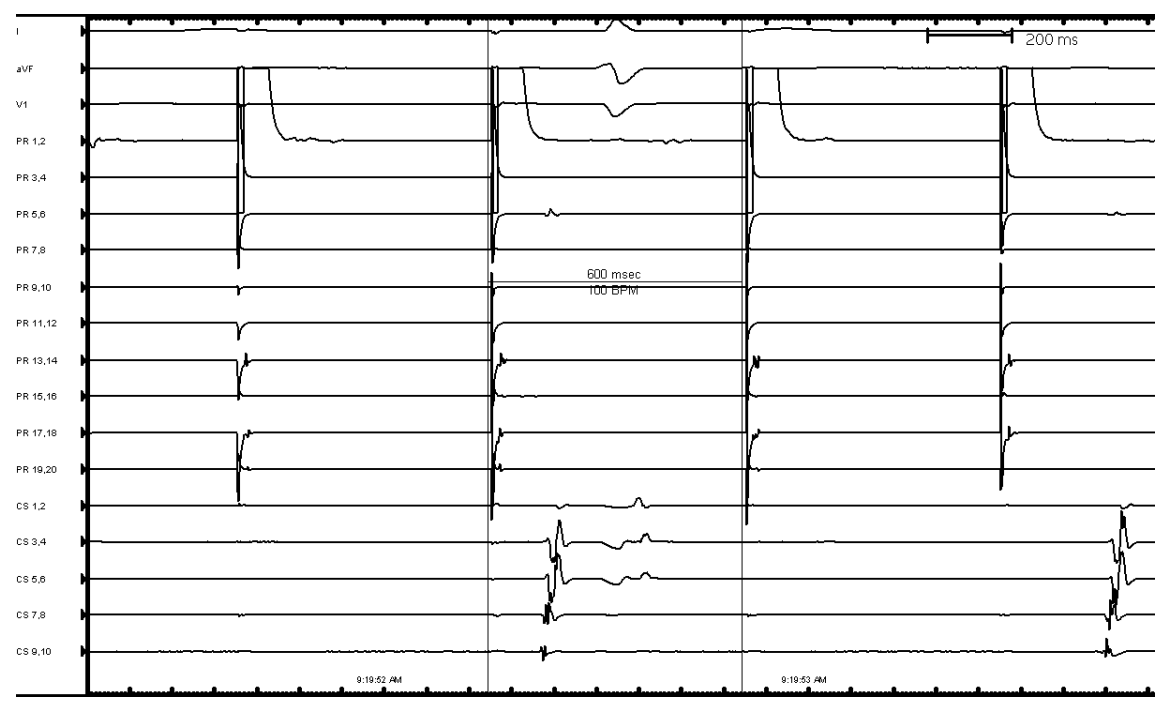

1. Haïssaguerre M, Jaïs P, Shah DC, et al. Spontaneous initiation of atrial fibrillation by ectopic beats originating in the pulmonary veins. N Engl J Med. 1998;339(10):659-666.

2. January CT, Wann LS, Alpert JS, et al. 2014 AHA/ACC/HRS guideline for the management of patients with atrial fibrillation: a report of the American College of Cardiology/American Heart Association Task Force on practice guidelines and the Heart Rhythm Society. Circulation.2014;130(23):e199-267.

3. Jacobson JT, Ciaccio EJ, Biviano AB, Whang W, Garan H. Bradycardia-dependent conduction block into pulmonary vein after isolation. Circ Arrhythm Electrophysiol. 2014;7(4):762-763.

4. Yagishita A, Gimbel JR, Arruda M. Rate-Dependent Exit Conduction Block From Pulmonary Vein to Left Atrium After Entrance Block: New Implications of Pacing Rate to Confirm Bidirectional Conduction Block. Circ Arrhythm Electrophysiol. 2016;9(6).

5. Gabriels J, Beldner S, Donnelly J, Willner J, Epstein LM, Patel A. Escape mapping to achieve bidirectional block: A case series.Pacing Clin Electrophysiol. 2019;42(4):470-473. 
6. El-Sherif N, Jalife J. Paroxysmal atrioventricular block: are phase 3 and phase 4 block mechanisms or misnomers? Heart Rhythm.2009;6(10):1514-1521. 